\title{
Growth of 2,2-Biimidazole-Based Nanorods on Mica Substrate
}

\author{
Mukhles Sowwan, ${ }^{1}$ Mohammad Abul Haj, ${ }^{2}$ Maryam Faroun, ${ }^{1}$ Zafer Hawash, ${ }^{1}$ \\ Jamal Ghabboun, ${ }^{1,3}$ Alaa Rida, ${ }^{1}$ Abeer Karmi, ${ }^{1}$ Wadie Sultan, ${ }^{2}$ and Ghaleb A. Husseini ${ }^{1,4}$
}

\author{
${ }^{1}$ The Nanotechnology Research laboratory, The Department of Materials Engineering, Faculty of Engineering, \\ Al-Quds University, P.O. Box 2002, East Jerusalem, Palestine \\ ${ }^{2}$ Department of Chemistry, Al-Quds University, East Jerusalem, Palestine \\ ${ }^{3}$ Department of Physics, Bethlehem University, Bethlehem, Palestine \\ ${ }^{4}$ The Department of Chemical Engineering, American University of Sharjah, Sharjah, UAE
}

Correspondence should be addressed to Mukhles Sowwan, muksowwan@gmail.com

Received 29 June 2010; Revised 11 October 2010; Accepted 5 November 2010

Academic Editor: Christian Brosseau

Copyright (C) 2010 Mukhles Sowwan et al. This is an open access article distributed under the Creative Commons Attribution License, which permits unrestricted use, distribution, and reproduction in any medium, provided the original work is properly cited.

The synthesis of a one-dimensional single-stranded helix using the crystallization of silver (I) nitrate and 2,2-biimidazole has promising potential for use in the area of nanotechnology mainly because of its unique electrical properties and its structural similarity to naturally occurring nucleic acids. In this study, we report a new method for the deposition and growth of 2,2biimidazole-based nanorods on mica substrates by employing a complex solution of silver nitrate (I) and 2,2-biimidazole. The morphology and electrical polarizability of the prepared nanorods are investigated by tapping mode atomic force microscopy (AFM) and noncontact electrostatic force microscopy (EFM). The experimental results show highly polarizable and singly separated nanorods oriented in three preponderant directions. In addition, we show that the active $\mathrm{K}^{+}$ions on the mica surface are required for the formation of these nanorods. Additionally, these potassium ions are a critical factor in controlling the nucleation and morphology of nanostructures.

\section{Introduction}

Recently, intensive efforts have been directed on developing supramolecular architectures via the self-assembly of metal complex components. Because of their potential applications in the fields of supramolecular chemistry, asymmetric catalysis, and nonlinear optical materials, novel designs of metal complexes exhibiting helical architectures are continuously emerging [1-8]. Helical structures are formed by wrapping molecular strands about a central axis and their orientation may be controlled by supramolecular interactions including hydrogen bonds, $\pi$-stacking or metal ion coordination $[9,10]$.

The neutral molecule 2,2-biimidazole (H2biim) (shown in Figure 1(a)) has attracted considerable attention in the design and synthesis of helical structures mainly due to the coordinative versatility of metal cations, for example, silver (I) [11-22]. The Ag-H2biim system is capable of adopting multiple coordinating modes and a variety of geometries according to its counter anion characteristics $[12,16,23]$. Hester et al. [12] showed that silver (I) nitrate and H2biim crystallize from an aqueous solution to afford a singlestranded helix, as evidence by X-ray crystallography. They found that this assembly is based on an approximate cis conformation of H2biim acting in concert with the preferred linear two-coordinate nature of the ligated silver (I) atom. Such a nanostructure has promising potential for use in the area of nanotechnology mainly because of its unique electrical properties and its structural similarity to naturally occurring nucleic acids $[12,23]$. In this study, we report a new method for the deposition and growth of 2,2-biimidazole-based nanorods on mica substrates by employing a complex solution of silver nitrate (I) and 2,2-biimidazole. The characteristics of these nanorods, including morphology and electrical polarizability, are investigated using atomic force microscopy (AFM) and electrostatic force microscopy (EFM). 


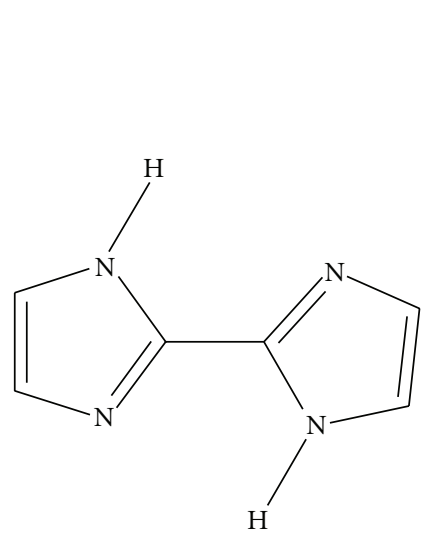

(a)

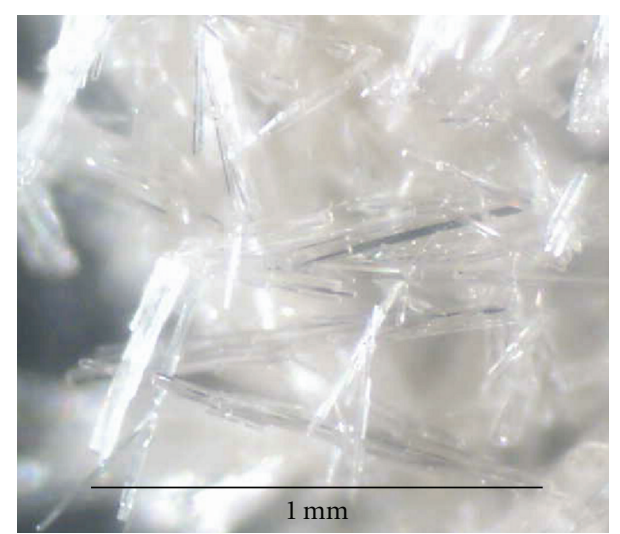

(b)

Figure 1: 2,2-biimedazole molecule and 2,2-biimedazole based columnar crystals (a) Schematics of 2,2 -biimedazole molecule. (b) Optical microscope image of $[\mathrm{Ag}(\mathrm{H} 2 \mathrm{biim})] \mathrm{NO}_{3}$ columnar crystals.

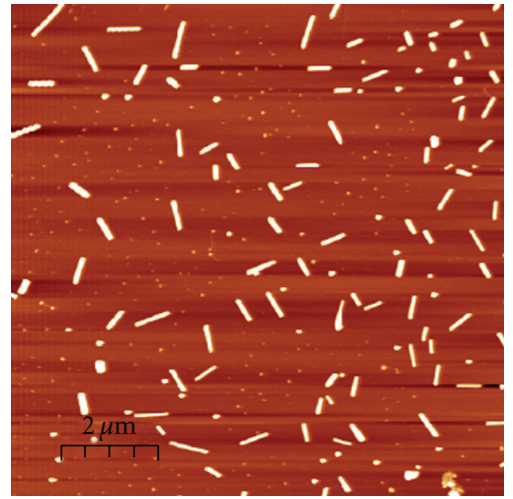

(a)

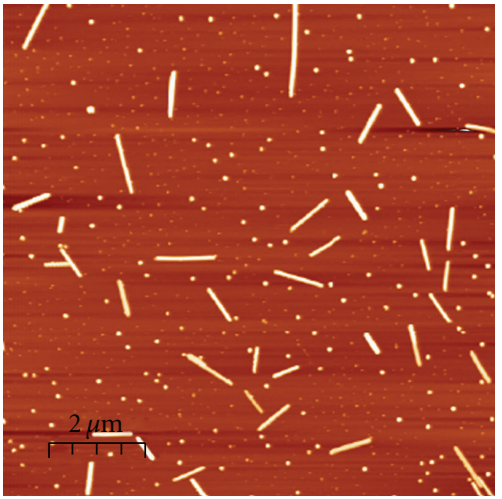

(b)

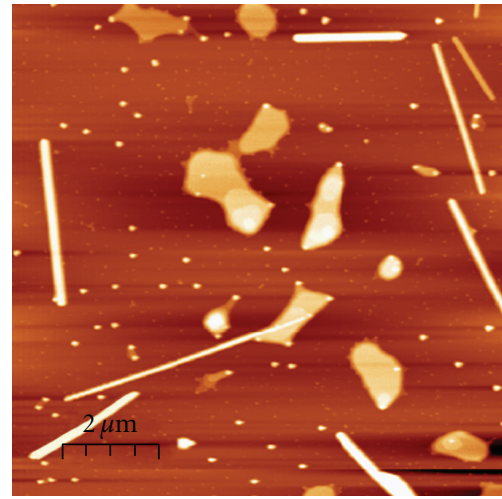

(c)

FIGURE 2: AFM topography images of 2,2-biimedazole-based nanorods on mica substrates at three deposition times (a) 10 min, (b) 20 min, and (c) $30 \mathrm{~min}$.

\section{Materials and Methods}

2.1. Materials. The muscovite mica used in the study possesses a layered structure formed using tetrahedraloctahedral-tetrahedral silicate sheets with a thickness of $1 \mathrm{~nm}$, separated by a monolayer of potassium atoms. The bonds between the silicate layers and the potassium ions are weak which allows for the easy cleavage of the mica and results in the formation of an atomically flat surface. Silver (I) nitrate, nitric acid and 2,2-biimidazole were purchased from Sigma Aldrich (St. Louis, MO, USA) and used as received.

\subsection{Growth and Deposition of Nanorods on Mica Surface.} One hundred thirty four $\mathrm{mg}$ of H2biim were dissolved in $20 \mathrm{~mL}$ of $0.01 \mathrm{M}$ aqueous solution of $\mathrm{HNO}_{3}$. Then, $340 \mathrm{mg}$ of $\mathrm{AgNO} 3$ were added as a solid to the above solution, resulting in the formation of a white precipitate. Hester et al. [12] reported that the precipitate can be resolubilized if the acidity of the solution is increased slowly. Columnar crystals are then deposited upon slow evaporation of the resulting solution, as shown in the optical microscope image of Figure 1(b). In this study, the columnar crystals obtained were resolubilized in a solution of $\mathrm{HNO}_{3}$ and of tripledistilled water at different concentrations. FTIR analysis of the deposited compound confirmed the results obtained previously by Hester et al. [12], showing the characteristic $\mathrm{N}-\mathrm{H}$ stretch $\left(3141-2810 \mathrm{~cm}^{-1}\right)$ of H2biim and the ionic nitrate absorption $\left(1360 \mathrm{~cm}^{-1}\right)$, indicating that the product is a complex involving both $\mathrm{AgNO}_{3}$ and $\mathrm{H} 2$ biim. Finally, a $10-\mu \mathrm{L}$ drop of the prepared solution was deposited on a mica substrate for different incubation times and dried with nitrogen gas.

2.3. AFM and EFM Imaging. AFM equipped with WSxM software (Nanotec S.L. Madrid, Spain) was used to characterize sample surfaces using the dynamic mode of AFM and EFM [24]. For all our silicon AFM probes, a Budget Sensor tips (Innovative Solutions Bulgaria Ltd., Sofia, Bulgaria) were used. These tips are electrically conductive with a chromium/platinum coating on both sides of the cantilever. The resonant frequency of the tips is $190 \mathrm{kHz}$ with a force constant of $48 \mathrm{~N} / \mathrm{m}$. EFM is extensively used as a noncontact 


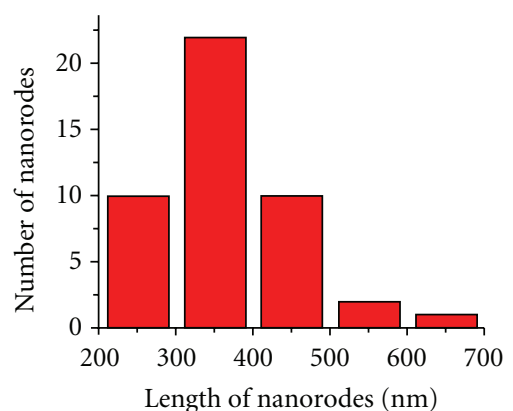

(a)

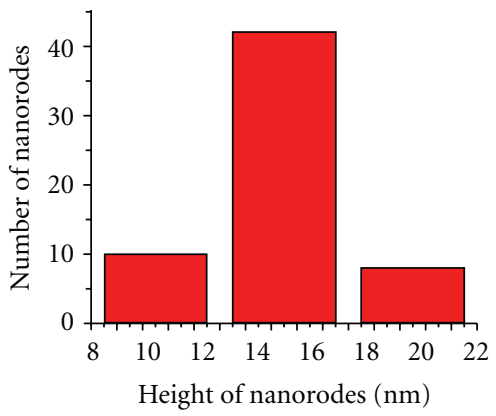

(d)

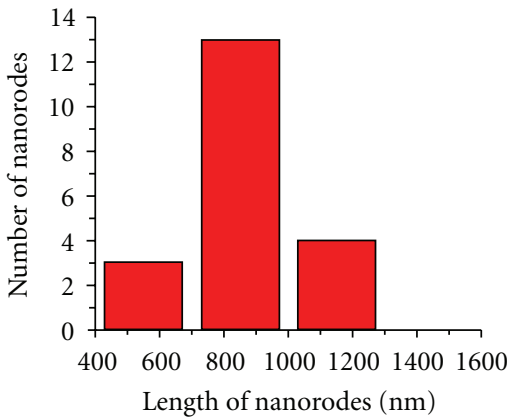

(b)

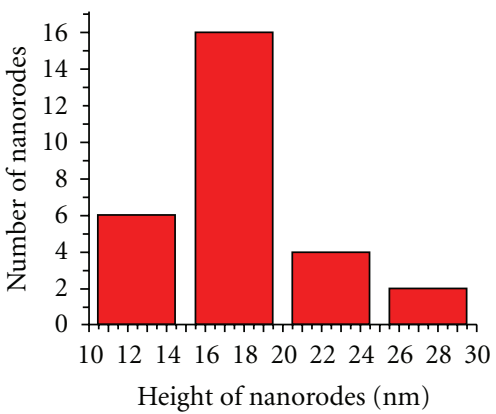

(e)

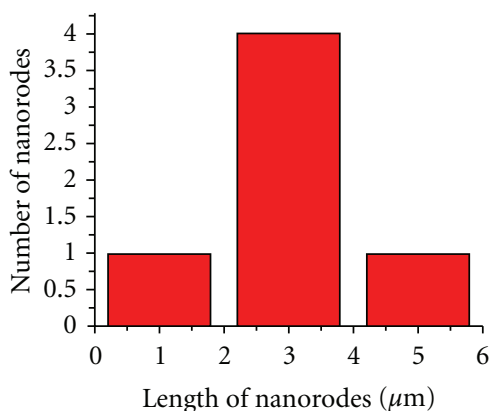

(c)

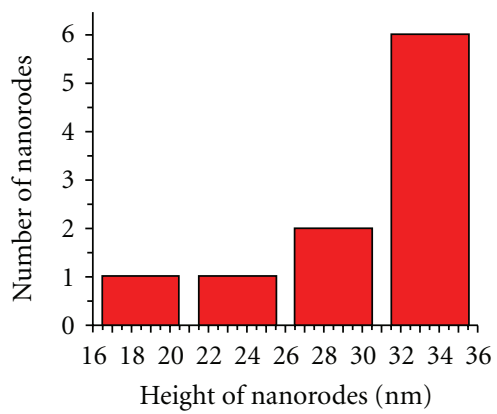

(f)

Figure 3: Analysis of the nanorods length and height by means of profile-sections associated with the AFM image of Figures 2(a), 2(b) and 2(c), respectively. The corresponding lengths vary in ranges (a) 200-700 nm, (b) 600-1200 nm, and (c) $1300-5000 \mathrm{~nm}$, while the corresponding heights vary in ranges (d) $11-21 \mathrm{~nm}$, (e) $11-27 \mathrm{~nm}$, and (f) $16-36 \mathrm{~nm}$ for deposition times of 10, 20, and 30 min, respectively.

and harmless method for polarizability detection of nanostructures on mica surfaces [25]. Retrace mode scanning was the main tool used in our study because it enabled the detection of an EFM signal by applying an electrostatic bias on the AFM tip. A topography scan was used, preceding the retrace scan, bearing the following EFM parameters: the bias, tip lift distance $(80 \mathrm{~nm})$ and the cancellation of the feedback. The dynamic amplitude was corrected to avoid any undesired tip deflections upon bias applications which could in turn lead to an erroneous reading of the phase shift imaging. A constant tip-sample distance was maintained during the retrace in the electrostatic force range. Additionally, when using the retrace scan, the feedback was canceled and a bias was applied simultaneously with a tip lift in the electrostatic range.

\section{Results and Discussion}

Figures 2(a), 2(b), and 2(c) are AFM topography images representing a typical feature of the nanorods grown on a mica surface with deposition times of 10,20, and $30 \mathrm{~min}$, respectively, using the resolubilized columnar crystal $(1 \mathrm{mg} / \mathrm{mL})$ of $0.01 \mathrm{M} \mathrm{HNO}_{3}$ solution. Most of the nanostructures in Figures 2(a), 2(b), and 2(c) are singly separated uniform nanorods.

Figures 3(a)-3(f) represent the profile analysis of the nanorods length and height associated with the AFM image for Figures 2(a), 2(b), and 2(c). It demonstrates that the longitudinal and cross-sectional lengths and heights are influenced by the deposition time $(t)$. The corresponding lengths vary in the range of $200-700 \mathrm{~nm}, 600-1200 \mathrm{~nm}$, and $1300-5000 \mathrm{~nm}$, while the corresponding heights vary in the range of $11-21 \mathrm{~nm}, 11-27 \mathrm{~nm}$, and $16-36 \mathrm{~nm}$ for the deposition times of 10,20 , and $30 \mathrm{~min}$, respectively. The nanoobject in the AFM topography image of Figure 4(a) (marked by a circle) suggests that the nanorod growth is initiated by the formation of a very thin and narrow rod. Then, it increases in thickness by the formation of another layer on the first nucleated rod. Figure 4(b) represents a magnification of the circled nanorod shown in Figure 4(a), and Figure 4(c) represents a longitudinal section showing the details of the layered structure of the nanorod. The rough surfaces of the nanorods observed in Figure 4(c) are possibly related to the release of solvent during the evaporation process. Nanorods grow with parallel orientations in three preponderant directions marked as 1,2, and 3 in the AFM topography images in Figure 4(a) and Figure S1 (see Figure S1 in the Supplementary Material available online at doi: 10.1155/2010706920) indicating strong interaction with the mica surface.

The surface of mica is negatively charged and hydrophilic due to the dissociation of the potassium cations from the mica surface. Previous studies reported that potassium ions in mica are easily replaced with other ions (such as $\mathrm{H}+$ ) when mica is treated with different solutions $[26,27]$. The ion exchange process is quick and efficient. In order to understand the role of $\mathrm{K}^{+}$ions present on the mica surface in the nanorods growth, two control experiments were performed, under the same conditions and a deposition time 


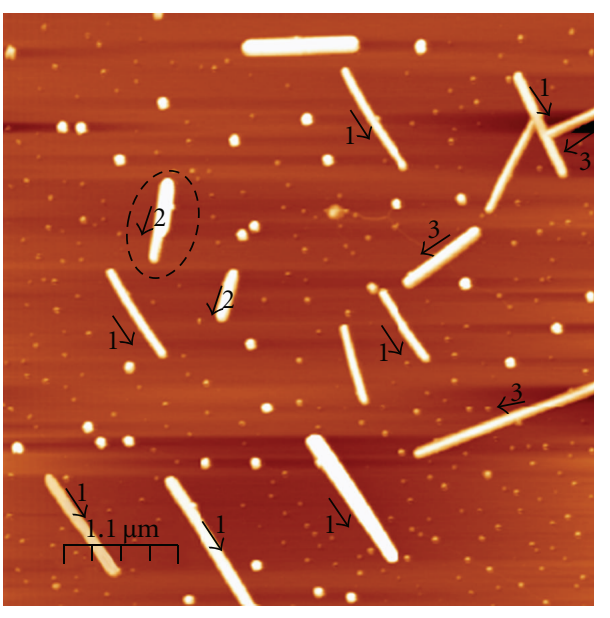

(a)

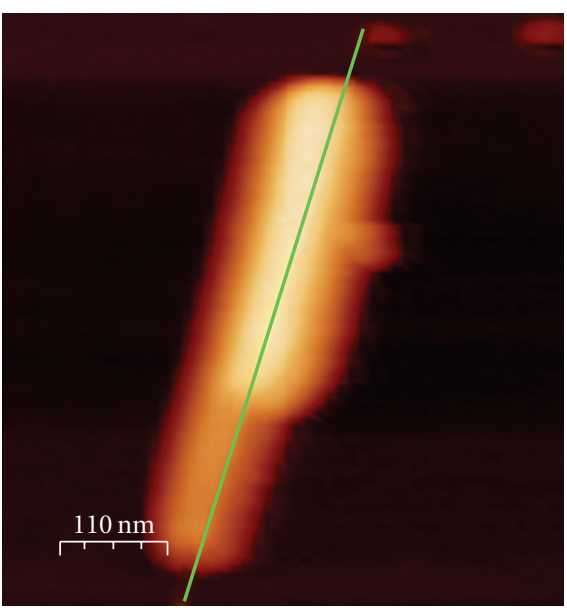

(b)

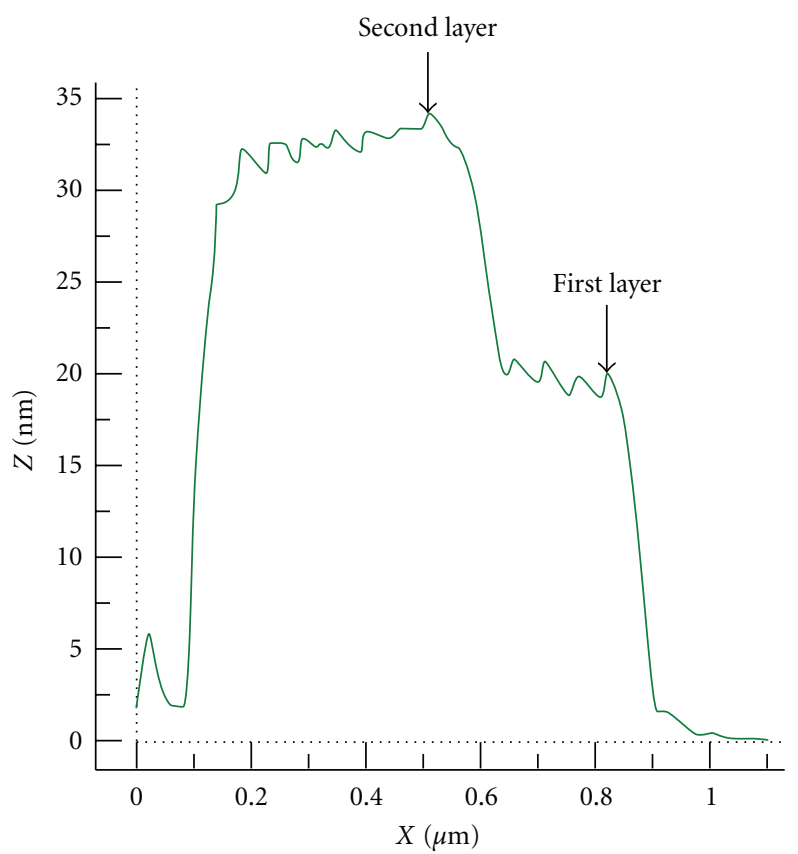

(c)

FIGURE 4: AFM topography images of 2,2-biimedazole-based nanorods on mica substrates. (a) The nanorods oriented in three preponderant directions marked $(1,2$, and 3$)$ on the image. (b) zoom of one of the nanorods marked by a circle in Figure 4(a), the image clearly shows the layered structure of the nanorod. (c) Longitudinal section of the nanorod in Figure 4(b) showing the two-layer (step) structure and the surface roughness of the nanorod.

of $10 \mathrm{~min}$, on two different substrates: a cleaved mica surface annealed at $350^{\circ} \mathrm{C}$ and ${\mathrm{a} \mathrm{SiO}_{2}}_{2}$ substrate. The surface of freshly cleaved mica is inherently negatively charged in water. The results showed the formation of nonhomogeneous three-dimensional aggregates. No nanorods were observed on the annealed mica and the $\mathrm{SiO}_{2}$ surfaces as shown in the AFM topography images of Figures S 2(a) and S 2(b), respectively. Therefore, we can deduce that the active $\mathrm{K}+$ ions on the mica surface are required for the formation of these nanorods. Additionally, these potassium ions are a critical factor in controlling the nucleation and morphology of nanostructures.
Upon increasing the concentration of the resolubilized columnar crystals to $10 \mathrm{mg} / \mathrm{mL}$ in the $0.01 \mathrm{M} \mathrm{HNO}_{3}$ solution, the grown structures exhibit ribbon-like morphology composed of parallel nanorods in a layered structure as presented in the AFM topography image of Figure 5. The nanostructures show no preference in orientation indicating a decrease in the morphological influence of the substrate.

The EFM measurements of the nanorods, shown in Figure 6(a), show a markedly interesting behavior. A strong pattern of the negative phase shift signal, where the nanorods were detected earlier using AFM, was observed when bias voltages of $+5 \mathrm{~V}$ (Figure 6(b)) and $-5 \mathrm{~V}$ (Figure 6(d)) were 


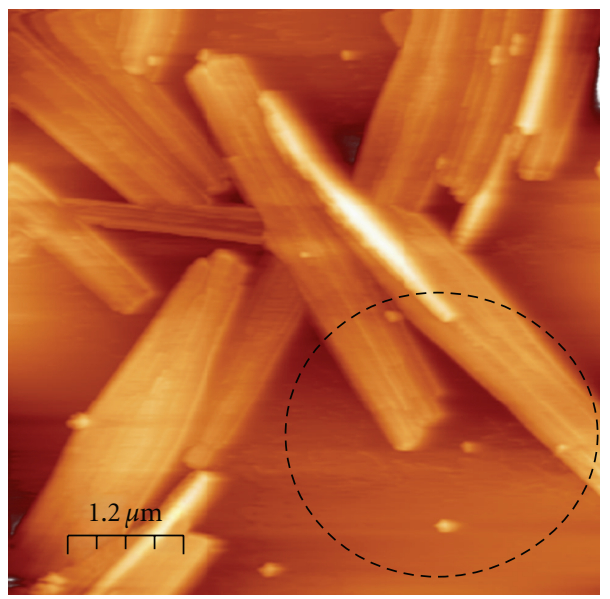

(a)

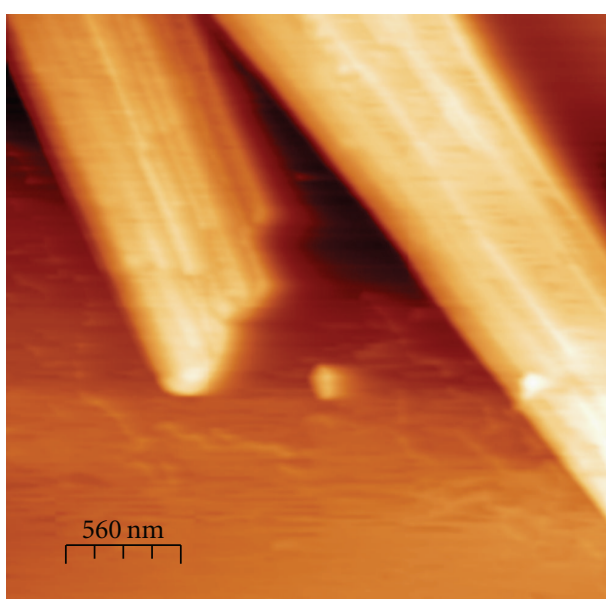

(b)

FIGURE 5: AFM topography image of the nanostructures deposited on mica from a high concentration of the resolubilized columnar crystals solution. (a) The grown structures exhibit ribbon-like morphology composed of parallel nanorods in a layered structure and show no preference in orientation indicating a decrease in the influence of the substrate. (b) Zoom of the area circled in (a).

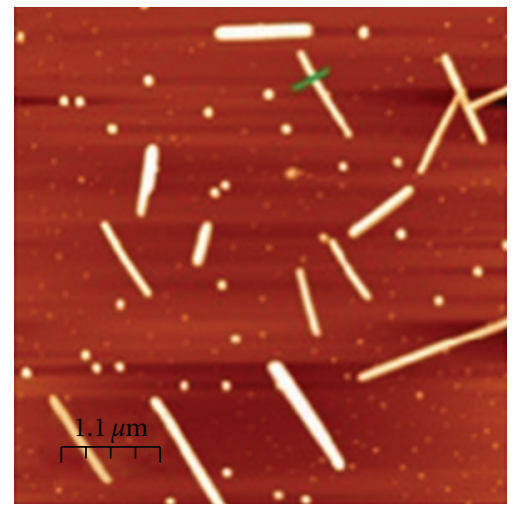

(a)

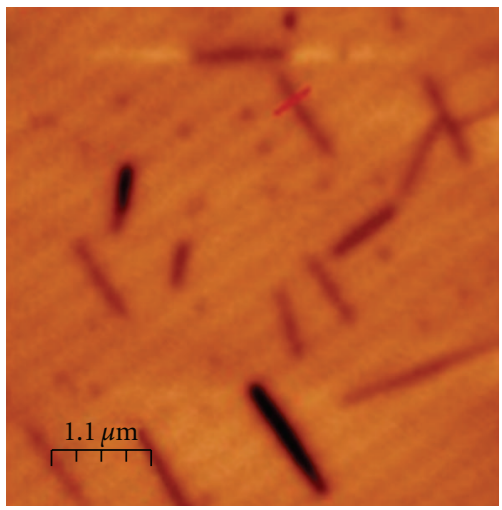

(d)

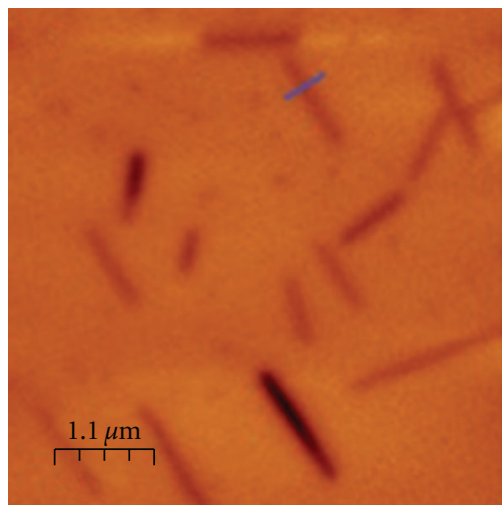

(b)

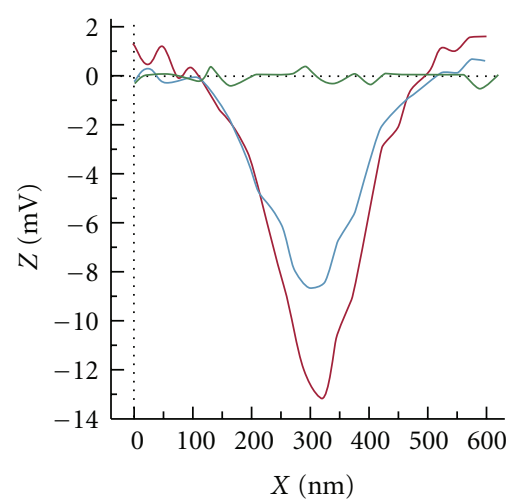

(e)

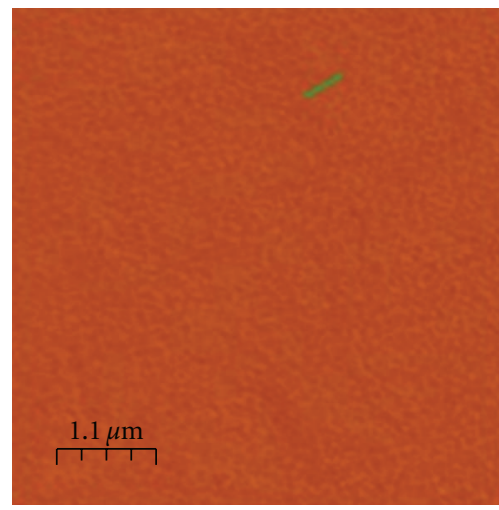

(c)

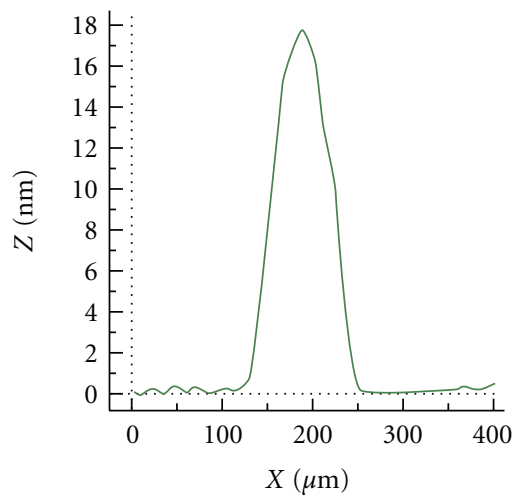

(f)

FIGURE 6: EFM image of 2,2-biimedazole-based nanorods. (a) Topography used for EFM imaging. (b) Phase imaging for a $+5 \mathrm{~V}$ applied tip bias and a lift of $80 \mathrm{~nm}$ from the surface. (c) Phase imaging for a $0 \mathrm{~V}$ applied bias with a tip lift of $80 \mathrm{~nm}$. (d) Phase imaging for a $-5 \mathrm{~V}$ applied bias with a tip lift of $80 \mathrm{~nm}$. (e) Cross-section diagram showing the intensity of the EFM signal through the phase imaging for molecules shown in (b), (c), and (d), follow colors. (f) Cross-section height profile of one nanorod shown in (a). 
applied to the tip, while no EFM signal was observed at $0 \mathrm{~V}$ (Figure 6(c)). The line profiles in Figure 6(e) represent the magnitude of the phase shift signal at the position of the nanorods using different bias voltages. This behavior is indicative of strongly enhanced polarizability which may be attributed to the H2biim moiety being coordinated to metal centers of silver (I).

In order to validate our results, we applied the same technique to multiwalled carbon nanotubes (MWCNTs), which are strongly polarizable objects comparable in height to the nanorods presented in this research (Figure S3 in Supplementary Materials). A strong pattern of a negative phase shift signal can clearly be observed at the position of the CNTs when bias voltages of $-5 \mathrm{~V}$ and $+5 \mathrm{~V}$ were applied to the tip, while no EFM signal was measured at $0 \mathrm{~V}$. The EFM results of CNTs are consistent with the results reported by other groups [25], supporting the reliability and accuracy of our EFM measurements.

\section{Conclusion}

We have successfully grown and deposited, nanometer scale rods from a solution containing a complex of $\mathrm{AgNO}_{3}$ and H2biim, on a mica substrate. Advanced AFM techniques were applied to characterize the morphological and electrical properties of these nanostructures. The experimental results revealed highly polarizable and singly separated nanorods oriented in three preponderant directions. The control experiments showed that the active $\mathrm{K}^{+}$ions on the mica surface were needed to form nanorods and are a critical factor in controlling the nucleation and the morphology of these nanostructures. These findings open the way for exploring the possibility of using these nano-objects in nanotechnology applications.

\section{Acknowledgments}

The Nanotechnology Research Laboratory is supported by the German Research Foundation (DFG), the French Ministry of Foreign Affairs, and Meyer Foundation.

\section{References}

[1] A. J. Sykes, Advances in Organic Chemistry: Toward the Construction of Functional Solid-State Supramolecular Metal Complexes Containing Copper (1) and Silver (1), vol. 46, Academic Press, Orlando, Fla, USA, 1999.

[2] M. J. Hannon and L. J. Childs, "Helices and helicates: beautiful supramolecular motifs with emerging applications," Supramolecular Chemistry, vol. 16, no. 1, pp. 7-22, 2004.

[3] C. Piguet, G. Bernardinelli, and G. Hopfgartner, "Helicates as versatile supramolecular complexes," Chemical Reviews, vol. 97, no. 6, pp. 2005-2062, 1997.

[4] M. Albrecht, "'Let's twist again"-double-stranded, triplestranded, and circular helicates," Chemical Reviews, vol. 101, no. 11, pp. 3457-3497, 2001.

[5] B. H. Ye, F. Xue, G. Q. Xue, L. N. Ji, and T. C. W. Mak, "Syntheses and characterization of two monomeric zinc complexes containing aqua ligands," Polyhedron, vol. 18, no. 12, pp. 1785-1790, 1999.
[6] W. J. Stratton and D. H. Busch, "The complexes of pyridinaldazine with iron(II) and nickel(II)," Journal of the American Chemical Society, vol. 80, no. 6, pp. 1286-1289, 1958.

[7] G. Morgan and F. H. Burstall, "Researches on residual affinity and co-ordination. Part XXXVII. Complex metallic salts containing 2:6-di-2' -pyridylpyridine $\left(2: 2^{\prime}: 2^{\prime \prime}\right.$-tripyridyl), Journal of the Chemical Society, pp. 1649-1655, 1937.

[8] E. C. Constable, S. M. Elder, M. J. Hannon, A. Martin, P. R. Raithby, and D. A. Tocher, " $2,2^{\prime}: 6^{\prime}, 2^{\prime \prime}: 6^{\prime \prime}, 2^{\prime \prime \prime}-$ Quaterpyridine (qtpy): a versatile ligand in metallosupramolecular chemistry; crystal and molecular structures of $\left[\mathrm{Ni}(\right.$ qtpy $\left.)\left(\mathrm{OH}_{2}\right)_{2}\right]\left[\mathrm{BF}_{4}\right]_{2}, \quad[\mathrm{Pd}($ qtpy $\left.)]\left[\mathrm{PF}_{6}\right]_{2}, \quad\left[\mathrm{Cu}_{2} \text { (qtpy }\right)_{2}\right]$ $\left[\mathrm{Pf}_{6}\right]_{2}$ and $\left[\mathrm{Ag}_{2}(\mathrm{qtpy})_{2}\right]\left[\mathrm{BF}_{4}\right]_{2}$," Journal of the Chemical Society Dalton Transactions, no. 12, pp. 2423-2433, 1996.

[9] S. J. Geib, C. Vicent, E. Fan, and A. D. Hamilton, "A self-assembling, hydrogen-bonded helix," Angewandte Chemie (International Edition in English), vol. 32, no. 1, pp. 119-121, 1993.

[10] V. Berl, I. Huc, R. G. Khoury, and J. M. Lehn, "Helical molecular programming: folding of oligopyridine-dicarboxamides into molecular single helices," Chemistry: A European Journal, vol. 7, no. 13, pp. 2798-2809, 2001.

[11] D. T. Cromer, R. R. Ryan, and C. B. Storm, "Structure of 2,2' biimidazole," Acta Crystallographica Section C, vol. 43, no. 7, pp. 1435-1437, 1987.

[12] C. A. Hester, R. G. Baughman, and H. L. Collier, "A simple helix based on 2,2' -biimidazole. Crystal and molecular structure of $\left[\mathrm{Ag}\left(\mathrm{NO}_{3}\right)\left(\mathrm{H}_{2} \text { biim }\right)\right]_{n}$," Polyhedron, vol. 16, no. 16, pp. 2893-2895, 1997.

[13] J. Cancela, M. J. González Garmendia, and M. Quirós, “Crystal structure, spectroscopy and magnetism of chlorobis $\left(2,2^{\prime}\right.$ biimidazole)oxovanadium(IV) chloride: the chloride anion as hydrogen bond acceptor forming infinite chains," Inorganica Chimica Acta, vol. 313, no. 1-2, pp. 156-159, 2001.

[14] R. Sang and LI. Xu, "Aquabis(2,2'-biimidazole)copper(II) dinitrate," Acta Crystallographica Section E, vol. 61, no. 4, pp. m793-m795, 2005.

[15] R. Sang and L. Xu, "A series of single, double, and triple $\mathrm{Me}_{2}$ biim-bridged dinuclear, trinuclear, and polymeric complexes: syntheses, crystal structures, and luminescent properties," Inorganic Chemistry, vol. 44, no. 10, pp. 3731-3737, 2005.

[16] M. Tadokoro, K. Isobe, H. Uekusa et al., "Cation-dependent formation of superstructures by one-pot self-organization of hydrogen-bonded nickel complexes," Angewandte Chemie International Edition, vol. 38, no. 1-2, pp. 95-98, 1999.

[17] M. Tadokoro, H. Kanno, T. Kitajima et al., "Self-organizing super-structures formed from hydrogen-bonded biimidazolate metal complexes," Proceedings of the National Academy of Sciences of the United States of America, vol. 99, no. 8, pp. 49504955, 2002.

[18] J. S. Casas, A. Castiñeiras, Y. Parajó et al., "Pd(II) and Pt(II) complexes of 2,2'-biimidazole and its $N^{\prime}$-dimethyl derivative. The crystal structure of $\left[\{\mathrm{PtBr}(\mathrm{DMSO})\}_{2}\left(\mathrm{Me}_{2} \mathrm{bim}\right)\right]$ $\left(\mathrm{Me}_{2}\right.$ bim=N'-dimethyl-2,2'-biimidazole)," Polyhedron, vol. 22, no. 8, pp. 1113-1121, 2003.

[19] B. J. Holliday and C. A. Mirkin, "Strategies for the construction of supramolecular compounds through coordination chemistry," Angewandte Chemie International Edition, vol. 40, no. 11, pp. 2023-2043, 2001.

[20] S. W. Kaiser, R. B. Saillant, W. M. Butler, and P. G. Rasmussen, "Rhodium and iridium complexes of biimidazole. 1. Mononuclear and dinuclear species," Inorganic Chemistry, vol. 15, no. 11, pp. 2681-2687, 1976. 
[21] A. Maiboroda, G. Rheinwald, and H. Lang, "Synthesis and Xray structure of the homobinuclear 2, 2' -biimidazole-bridged palladium(II) complex $[\{\operatorname{Pd}(\mathrm{dppp})\}(\mu$-biim $)](\mathrm{OTf})_{2}$, Inorganic Chemistry Communications, vol. 4, no. 8, pp. 381-383, 2001.

[22] P. Majumdar, S. M. Peng, and S. Goswami, "Biimidazole complexes of $\mathrm{ML}_{2}{ }^{2+}[\mathrm{M}=\mathrm{Ru}$ or Os, $\mathrm{L}=2$-(phenylazo)pyridine $]$. Synthesis, structure and redox properties of mono- and dinuclear complexes

," Journal of the Chemical Society Dalton Transactions, no. 10, pp. 1569-1574, 1998.

[23] R. L. Sang and LI. Xu, "Counteranion-induced formation of cis and trans singly and doubly $\mathrm{H}_{2}$ biim-bridged di-, hexa, and polymeric Ag- $\mathrm{H}_{2}$ biim complexes," European Journal of Inorganic Chemistry, no. 6, pp. 1260-1267, 2006.

[24] I. Horcas, R. Fernández, J. M. Gómez-Rodríguez, J. Colchero, J. Gómez-Herrero, and A. M. Baro, "WSXmml: a software for scanning probe microscopy and a tool for nanotechnology," Review of Scientific Instruments, vol. 78, no. 1, Article ID 013705, 2007.

[25] A. Bachtold, M. S. Fuhrer, S. Plyasunov et al., "Scanned probe microscopy of electronic transport in carbon nanotubes," Physical Review Letters, vol. 84, no. 26, pp. 6082-6085, 2000.

[26] L. Xu and M. Salmeron, "Effects of surface ions on the friction and adhesion properties of mica," Langmuir, vol. 14, no. 8, pp. 2187-2190, 1998.

[27] D. E. Dunstan, "Forces between mica surfaces in $\mathrm{CaClS}_{2}$ solutions," Langmuir, vol. 8, no. 2, pp. 740-743, 1992. 

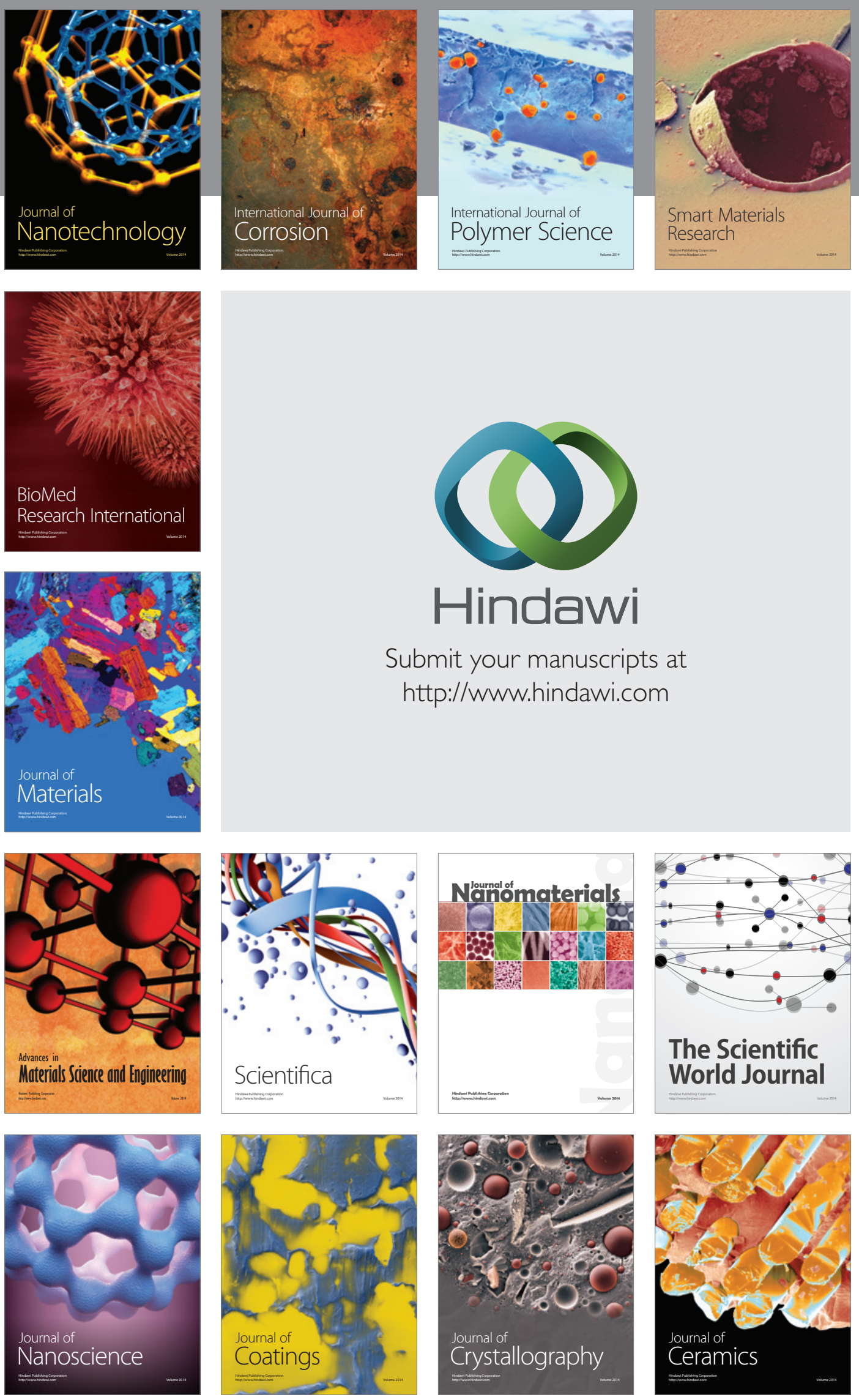

The Scientific World Journal

Submit your manuscripts at

http://www.hindawi.com

\section{World Journal}

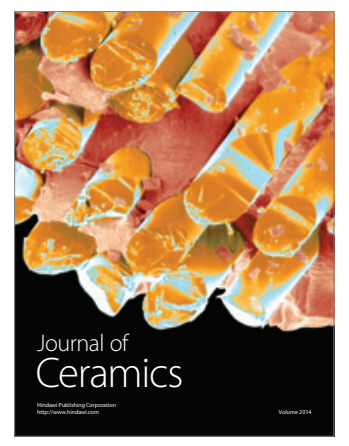

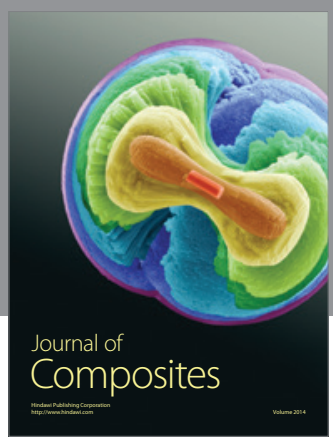
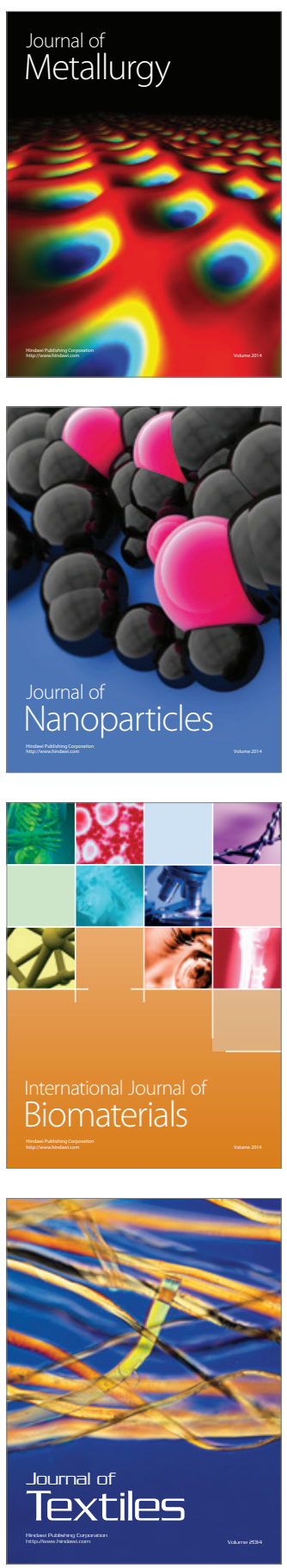